scanning the literature within one's own fields of interest, and to dip into and find nuggets of fascination which prompt a further exploration of the field.

This is not a book for junior medical staff to buy, but it is a book for those with enquiring and research-oriented minds to search out in the local medical library to stimulate further reading and research activities.

\title{
COLIN ROBERTSON
}

Department of Accident \& Emergency Medicine,

Royal Infirmary,

Edinburgh, Scotland

\section{Essential Radiology in Head Injury-A Diagnostic Atlas of Skull Trauma}

By D. W. H. MOK \& L. KREEL

Heinemann Medical Books, Oxford, 1988. 209 pp., 203 figs., $£ 35.00$.

Correct management of patients with head injuries is one of the most difficult challenges $ᄋ$ faced by inexperienced casualty officers. This book is an attempt to cover the relevant portions of several standard radiological texts in one volume of reasonable size. In this endeavour the joint authors, one an orthopaedic surgeon and the other a radiologist of international repute, have succeeded.

A short introduction deals with the Royal College of Radiologists' selection criteria for skull radiography following trauma, supplemented by the authors' own views Chapters on radiographic positioning and radiological anatomy follow and then the meat of the book is given over to the diagnosis of fractures and their associated featureș Illustrations of fractures are interspaced by normal variants which simulate traumatic lesions. This mixing of 'lesions' and 'non-lesions' makes the book difficult to use as a quick reference in the middle of the night, but a more authorititive text (Keats, 1988) is available for this purpose.

Further chapters cover the special features of paediatric fractures, basal fractures, post-surgical appearances and foreign bodies. Probably the best-written and most valuable section of the book is that given over to facial injuries. Several CAT scans are included for completeness as our projections such as the submentovertical which are unsuited to the casualty department. Each chapter has a short list of references and suggested further reading.

There are the usual number of proof-reading errors and rather vague anotations of illustrations, but these are of a minor nature. The scope of this book is wide and the authors are to be congratulated on producing a concise and readable book (perhaps the page demonstrating poor quality films, together with rather trite comments, could have been omitted!).

At the end of the day - when indeed this work will be of most value to its intended user-a book illustrating radiographs succeeds or fails on its standard of image reproduction. With only a handful of exceptions the 203 illustrations are of at least acceptable quality.

In summary I would recommend this reasonably priced book to all new casualty officers as an excellent guide to the radiological management of head injuries. Mutual 
benefit is to be gained both for the patient and the doctor, particularly in the 'wee small hours'.
A. W. HORROCKS
Consultant Radiologist,
Wythenshawe Hospital,
Manchester, England

\section{REFERENCE}

Keats T. (1988) Atlas of normal roentgen variants that may simulate disease 4th edn. Wolfe year book.

\section{Clinical and Resuscitative Data}

By R. P. H. Dunhill \& M. P. Colvin

Blackwell Scientific Publications, Oxford, 1989. 248 pp. $£ 16.95$.

This is the fourth edition of a book with which I must admit to be unfamiliar. It has been intended to provide essential data and information to assist doctors in resuscitation. It is apparently aimed particularly at the accident and emergency department.

There are many old chestnuts in the book which detract from its usefulness. For example, the calculation of correction of metabolic acidosis by the base deficit $\times \frac{1}{3}$ of the weight in $\mathrm{kg}$ to give you the mmol of bicarbonate is not helpful in many resuscitation episodes and for example after cardiac arrest this would now be considered totally inappropriate. Such isolated information without background discussion may mislead the inexperienced. However, there are many nuggets of information particularly related to anaesthesia which I found extremely useful. I was pleasantly surprised to find such a good section on local and regional anaesthesia with some excellent illustrations.

When the book strayed into critical care resuscitation it met some fundamental problems. For assessing and resuscitating patients with multiple injuries there are welldocumented tried-and-tested protocols. Any modern text must now refer to ATLS. The personal approach of the authors could not be recommended in many modern accident and emergency departments, including my own. There is little practical help to be found and some potentially misleading unqualified advice. The administration of inotropic agents without the measurement of cardiac output and systemic vascular resistance is not the best practice-to advise this in the seriously injured cannot be justified.

If the section on major trauma is unhelpful then the section on cardiac arrest is worse. The Resuscitation Council have produced clear guidelines based on current European and North American consensus and if followed prevent idiosyncratic and unjustifiable recommendations, as $150 \mathrm{mEq}$ of bicarbonate at the start of cardiac arrest.

It is such a pity that the section on critical care is so out-of-date when there is so much useful information in this book. The chart on dosage of drugs delivered by infusion is almost worth buying the book for. The section on critical care must be put in context 